\title{
Rehabilitation of medical personnel as an actual problem
}

\section{Opinion}

Medical personnel, during its professional activities, face the adverse effects of a significant number of professional factors: mechanical, physical, chemical, psycho-emotional. These factors affect the level of health of doctors and nurses, contributing (among other things) to the development of occupational diseases. According to the level of occupational pathology, medical workers occupy the fifth place among representatives of all professional groups. Our study, which covered 650 nurses and 200 doctors in various regions of Russia, revealed serious changes in their health status. So, according to self-assessment data, $37.0 \%$ of the patients had chronic diseases. The proportion of those who have them increases from $18.4 \%$ under the age of 30 to $44.4 \%$ at the age of 50 and older. The most frequently noted diseases were: pathology of the musculoskeletal system (available in 44.4\%); cardio-vascular system (33.3\%); allergic pathology $(22.2 \%)$. Along with somatic diseases, there were psycho-emotional disorders, in particular, fatigue phenomena $(83.3$ per 100 respondents), irritability (61.1\%) and autonomic disorders (44.4\%). $83.2 \%$ of those surveyed over the age of 40 noted that the usual situations at work make them irritable. Only $45.2 \%$ of medical workers believed that they had a calm sufficient in depth and duration of sleep. A study using a special method for the presence of emotional burnout syndrome showed that $36.7 \%$ of the examined patients do not have it, in $33.3 \%$ of cases it is formed, in $30.0 \%$-have formed (among the persons over 55 years old-in 55.6\%). The quality of life level (estimated using the SF-36 questionnaire) was generally reduced by one third and amounted to 66.7 .

Special studies show that with a properly organized system of workers' health protection, it is possible to reduce their incidence by $40-50 \%$. If in the pre-perestroika period the system of Soviet health provided for the existence of special medical organizations (units), whose purpose was to protect the health of the working population, then their number fell sharply. Modern legislation provides for the right of employers to create such units, but this is not their responsibility. However, there is experience in the functioning of special units in medical organizations to strengthen the health of medical workers. But in general, there is no system of prevention and rehabilitation among them. Considering the urgency of the problem, we consider it as an important task for health managers to develop appropriate programs in all medical organizations with the definition of specific performers, organizational structures, methods and targets. At the same time, existing capacities of medical organizations (physiotherapy, mechanotherapy, physiotherapy departments, swimming pools, halo

\author{
Volume 2 Issue 4 - 2017
}

\section{Petrova NG, Pogosyan SG, Minnullin TI, Bratslavsky VB \\ Department of the Nursing Care, Saint Petersburg State} Medical University, Russia

Correspondence: Petrova Natalia G, Department of the Nursing Care, Saint Petersburg State Medical University, Russia, Email petrova-nataliya@bk.ru

Received: November 25, 2017| Published: December II, 2017

cameras, psychological relief rooms, etc.) can be actively used for these goals. Obligatory, in our opinion, should be the presence in the staff of the organization of a psychologist who would conduct individual and group trainings with the staff, teach it the methods of autorelaxation. The tasks of such service should include the formation of workplace of all categories of medical personnel, taking into account the requirements of ergonomics, providing nurses with means of small mechanization. For the purposes of recovery and rehabilitation, as well as the formation of the corporate spirit and the strengthening of relationships in the collectives, mass health-improving activities (departure to nature, sports competitions) and cultural and leisure activities should be regularly conducted. We consider the use of halo chambers, oxygen cocktails, phytotherapy, detensor therapy, music therapy and psycho-relaxation techniques ("progressive relaxation" with alternating tension and relaxation of individual muscle groups) to be the most accessible from specific health procedures. Important aspects of the rehabilitative impact should be: a mentoring system, a clear and transparent system of incentives, career development of employees. In the future, it is possible to differentiate financial incentives for employees depending on their level of health and active participation in rehabilitation programs. Rehabilitation of medical personnel and strengthening of its health will also contribute to improving the quality of medical care.

\section{Acknowledgements}

None.

\section{Conflict of interest}

There is no conflict of interest in composing this manuscript. 